\title{
Global Optimization with Electro Weak
}

\author{
Z. Emami ${ }^{1 *}$, M. H. Farahi ${ }^{2}$ and E. Ansari ${ }^{2}$ \\ ${ }^{I}$ Department of Physics, School of Sciences, Islamic Azad University, Mashhad Branch, Iran \\ ${ }^{2}$ Department of Mathematics, School of Sciences. Islamic Azad University, Mashhad Branch, Iran
}

\begin{abstract}
Mathematician tried to improve the existing methods by generating new methods being able to increase the preciseness of results.

This paper introduces a new heuristic method for global optimization. The method utilizes an attraction -repulsion mechanism to move the sample points towards the optimality. The method is in fact a kind of modification of the so-called Electromagnetic method, but it is more flexible than Electromagnetic. The proposed scheme can be used either as a uniqe approach or as an accompanying procedure for other methods.

The used mechanism, included, a combination methods of electromagnetic like (EM) heuristic algorithm, with attraction- repulsion force, to move the sample point from feasible region toward optimization, then with using the Nelder-Mead heuristic searching method, the former points in (EM), converges toward global optimization.
\end{abstract}

Keywords: Global optimization, Nelder Mead simplex method, Feasible region, Test functions, Electromagnetic method, Electro weak method.

\section{INTRODUCTION}

To days applications of important mathematical methods in different disciplines developed more than before .Then for achieving optimization new approaches developed. Including to these, one should mentioned the global optimization that could be used for solving problems in mathematics, physics, chemistry, industry and economics.

In most problems in the fields, the special features as discontinuity, non differentiability and or non reparability of the functions etc... introduced which, formal mathematical process with key roles as gradient process is not usable especially with lots of variable in hand and the uncertainty of the achievements [1-6].

To reduce all these, and reaching better response with high accuracy we devised an applicable method for global optimization, named Electro Weak (EW) method.

The privileges of the method are as bellow:

1. Increasing the calculations accuracy.

2. Increasing the convergence acceleration (shortening the calculation time).

3. Vast applicability of the method [7,8] for using the global optimization.

\section{ELECTRO WEAK (EW) HEURISTIC METHOD- OLOGY}

Based on this new method we reach to the following problem:

\footnotetext{
*Address correspondence to this author at the School of Sciences, Physics Dept, Islamic Azad University, Mashhad Branch, Iran;

Tel: +985118435000; Fax : +985118424020;

E-mail: zahra_sh_emami@yahoo.com

This work supported financially by research dean of the Islamic Azad University Mashhad branch (89243/301/4).
}

$\operatorname{minimize} f(\mathrm{x})$

s. $t . x \in S$

$S=[l, u]=\left\{x \in R^{n} \mid l_{k} \leq x_{k} \leq u_{k}, l_{k}, u_{k} \in R, k=1,2, \ldots, n\right\}$

It assumed that the following quantities are at hand:

$\mathrm{n}$ : the problem dimension

$\mathrm{f}(\mathrm{x})$ : the objective function

$\mathrm{u}_{\mathrm{k}}$ : upper limit in $\mathrm{k}^{\mathrm{th}}$ dimension

$\mathrm{l}_{\mathrm{k}}$ : lower limit in $\mathrm{k}^{\text {th }}$ dimension

The EW method is heuristic and stochastic one because it starts from feasible region with stochastic sampling tend to global optimization of the problem (see $[9,10]$ for more details). In fact the EW global optimization method is a combination of two Nelder- Mead (NM) and Electromagnetismlike Mechanism (EM) plus a new excess trend, that leads to obtain global optimization including rather all test problem with high accuracy and fast convergence of the achievements.

Here we introduce (NM) briefly where for more details one can see for example [11-13].

The Nelder -Mead simplex algorithm is the most widely used direct search method for solving the unconstrained optimization problem.

$\min f(x)$

where $f: R^{n} \rightarrow R$ is called the objective function and $\mathrm{n}$ the dimension. A simplex is a geometric figure in $\mathrm{n}$ dimensions that is the convex hull of $n+1$ vertices. We denote a simplex with vertices $x_{1}, x_{2}, x_{3}, \ldots, x_{n+1}$ by $\Delta$. 
The Nelder-Mead method iteratively generates a sequence of simplices to approximate an optimal point of (2). At each iteration the vertices of the simplex are ordered according to the objective function values.

$f\left(x_{1}\right) \leq f\left(x_{2}\right) \leq \cdots \leq f\left(x_{n+1}\right)$

The algorithm uses four possible operations: reflection, expansion, contraction, and shrink, each being associated with a scalar parameter: $\alpha$ (reflection), $\beta$ (expansion), $\gamma$ (contraction), and $\delta$ (shrink). The values of these parameters satisfy $\alpha>0, \beta>1,0<\gamma<1$, and $0<\delta<1$. In the standard implementation of the Nelder -Mead method for example, the parameters are chosen to be $\{\alpha, \beta, \gamma, \delta\}=\{1,2,1 / 2,1 / 2\}$.

Let $x$ be the centroid of the $n$ best vertices.

Now one may outlines the Nelder-Mead method as follows (see [11, 12]):

In one iteration of the Nelder-Mead algorithm :

1. Sort. Evaluate $\mathrm{f}$ at the $n+1$ vertices of $\Delta$ and sort the vertices so that (3) holds.

2. Reflection. Compute the reflection point $x_{r}$ from $x_{r}=x_{0}$ $+\alpha\left(x-x_{n+1}\right)$.

Evaluate $f_{r}=f\left(x_{r}\right)$. If $f_{1} \leq f_{r}<f_{n}$, replace $x_{n+1}$ with $x_{r}$.

3. Expansion. If $f_{r}<f_{l}$ then compute the expansion point $x_{e}$ from

$x_{e}=. \mathrm{x}+\beta\left(x_{r}-. x\right)$ and evaluate $f_{e}=f\left(x_{e}\right)$. If $f_{e}<f_{r}$, replace $x_{n+1}$ with $x_{e}$; otherwise replace $x_{n+1}$ with $x_{r}$.

4. Outside Contraction. If $f_{n} \leq f_{r}<f_{n+1}$, compute the outside contraction point $x_{o c}=. x+\gamma\left(x_{r}-. x\right)$ and evaluate $f_{o c}=f$ $\left(x_{o c}\right)$. If $f_{o c} \leq f_{r}$, replace $x_{n+1}$ with $x_{o c}$; otherwise go to step 6 .

5. Inside Contraction. If $f_{r} \geq f_{n+1}$, compute the inside contraction point $x_{i c}$ from $x_{i c}=. x-\gamma\left(x_{r}-. x\right)$ and evaluate $f_{i c}=f$ $\left(x_{i c}\right)$. If $f_{i c}<f_{n+1}$, replace $x_{n+1}$ with $x_{i c}$; otherwise, go to step 6 .

6. Shrink. For $2 \leq i \leq n+1$, define $x_{i}=x_{1}+\delta\left(x_{i}-x_{1}\right)$.

Although lacking a satisfactory convergence theory, the Nelder-Mead method generally performs well for solving small dimensional real life problems and continuously remains as one of the most popular direct search methods [11, 12].

It has been observed by many researchers, however, that the Nelder-Mead method can become very inefficient for large dimensional problems (see, for example, [12] and its references).

Now the general scheme for (EM) is discussed briefly. In fact (EM) consists of four phases. These are initialization of the algorithm, calculation of the total force exerted on each point (as a particle), movement along the direction of the force, and application of the neighborhood search to exploit the local minima of the function $f(x)$. The general scheme can be seen in [9].

As mentioned earlier, the (EW) method is a combination of two so-called methods.

To do so, first with using heuristic (EM) method and random sampling the best point $\left(\mathrm{x}_{\text {best }}\right)$ will identified, this in turn will be the primary point of (NM) method. The lower and upper limits of the variable are defined with $l_{k}$ and $u_{k}$ respectively.

The initial simplex selection would lead to global optimized responses continuously. The Algorithm (1) shows this.

In this algorithm we have:

$\mathrm{m}$ : number of sample points.

MAXITER: maximum algorithm iteration.

LSITER: minimum algorithm iteration in global search.

$\delta$ : Local search parameter so as

$\delta \in[0,1]$

Algorithm (1)

$E W\{m, f, L, U, M A X I T E R, L S I T E R, \delta\}$

1. $\left[x_{\text {best }}, X, f\right]=\operatorname{Initialize}(m, z, L, U)$

2.iteration $\leftarrow 1$

3. while iteration $<$ MAXITER do

4. $\left[x_{\text {best }}, f_{b}, E\right]=\operatorname{Local}(L, U, \operatorname{LSITER}, \delta, z, X, m, f)$

5. $F=\operatorname{CalcF}\left(F, f_{b}, x_{\text {best }}, z, X, m, E\right)$

6. $X=\operatorname{Move}(F, X, L, U$, best,$m)$

7. iteration $\leftarrow$ iteration +1

8.end while

9. $\left[B, f_{b}\right]=N M\left(x_{\text {best }}, L, U, z\right)$

10. $B, f_{b}$

The initial sub program for sampling $\mathrm{m}$ random point from feasible region is used from hyper cube.

It assumed that sample points distributed between upper and lower bound homogeneously. This program will ended when $\mathrm{m}$ points together with the $\mathrm{x}_{\text {best }}$ have the best objective function. The goal of the sub program in other hand is collecting local information from the point xi. LSITER and $\delta$ parameters of the program for iteration and neighboring search coefficient. It should be mentioned that each iteration acts as the foot step for progressing the sample points towards global optimization.

Obviously, the displacement of the footsteps in turn would led to the movement extension towards the optimization direction of the problem. That is in each iteration each selected point replaces, the neighboring i-th point until the best point $\mathrm{x}_{\text {best }}$ of the point reached. Noticeably the (EM) and (EW) approach are based upon, attraction- repulsion mechanism of electromagnetic- like relationship so that each sample point assumed as though there is a free charged particle pretending the goal function there.

If $\mathrm{i}$-th point charge is $\mathrm{q}_{\mathrm{i}}$, then from electrostatic we have $[9,10]$; 
Table 1. (EW 1) Program with $\varepsilon \circ=8.9 \times 10^{-12}$

\begin{tabular}{|c|c|c|c|}
\hline $\mathbf{f}(\mathbf{x})($ Optimized $)$ & $\mathbf{f}(\mathbf{x})($ best $)$ & X best & Test Function \\
\hline \hline 0.0000 & 0.0096 & $(1.0263,0.0145)$ & Complex \\
\hline 0.0000 & 1.7696 & $(1.3157,2.8363)$ & Davis \\
\hline 463.0000 & 463.7006 & $(1.8367,-6.0240)$ & E1 \\
\hline 0.0000 & 1.5517 & $(-10.2530,9.3149)$ & Himmelblau \\
\hline 0.0000 & 1.3293 & $(0.3351,1.3080)$ & Rastrigin \\
\hline-2.0000 & -1.5010 & $(1.1357,6.8603)$ & Rosenbrock \\
\hline 0.0000 & 0.1518 & $(12.1000,5.7234)$ & Spiky \\
\hline-38.85 & -38.7036 & $(-0.0995,-0.0411)$ & Stenger \\
\hline 0.0000 & 0.0317 & & \\
\hline
\end{tabular}

$q_{i}=\exp \left((-n) \frac{f(x i)-f\left(x_{\text {best }}\right)}{\sum_{k=1}^{m}\left(f\left(x_{k}\right)-f\left(x_{\text {best }}\right)\right.}\right)_{i=1 \ldots . m}$

It is noticeable that in heuristic (EW) method, the electric charge for each point will changes in every iteration. The point with best objective function have the most favorite electric charge. Calculation of the electric charge of each point in above mentioned manner, present the moving direction towards optimization in the next iteration. Total force applying to the $\mathrm{i}$-th point is $\mathrm{F}_{\mathrm{i}}$ as the superposition of (EM) long range force and short range of (EW) force. The comparison criteria for this is:

$d=\left(\sum_{k=1}^{n}\left(l_{k}-u_{k}\right)\right)^{\frac{1}{2}}$

For (EW) force the criteria is: $\left\|x_{i}-x_{j}\right\| \leq d / 5$ where the norm is Euclidian,

For $\mathrm{F}_{\mathrm{i}}$ we have:

$F_{i}= \begin{cases}\sum_{\substack{j=1 \\ j \neq i}}^{m}\left(x_{j}-x_{i}\right) \frac{q_{i} q_{j} e^{-\alpha\left\|x_{j}-x_{i}\right\|}}{\left\|x_{i}-x_{j}\right\|^{2}} & f\left(x_{j}\right)<f\left(x_{i}\right) \\ \sum_{\substack{j=1 \\ j \neq i}}^{m}\left(x_{i}-x_{j}\right) \frac{q_{i} q_{j} e^{-\alpha\left\|x_{j}-x_{i}\right\|}}{\left\|x_{j}-x_{i}\right\|^{2}} & f\left(x_{j}\right) \geq f\left(x_{i}\right)\end{cases}$

Here $\alpha$ is a parameter comprised the optimization of test function, selected with best fitted condition of $F_{i}$ that in our (EW) method is about 3.25. It should be mentioned that for long range points the $F_{i}$ force obtain refer to the following electromagnetic relationship as;

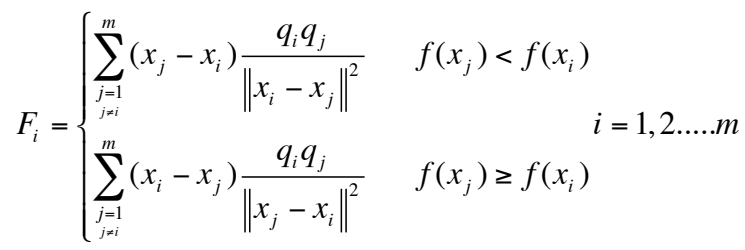

With total force $F_{i}$ the $\mathrm{i}$-th point moves randomly in the force direction or:

$x_{i} \leftarrow x_{i}+\lambda \frac{F_{i}}{\left\|F_{i}\right\|}(R N G)_{i=1,2 \ldots \ldots . m}$

The $\mathrm{x}_{\mathrm{i}}$ moves toward upper bound $\left(\mathrm{u}_{\mathrm{k}}\right)$ or lower bound $\left(l_{k}\right)$. Apparently the $x_{\text {best }}$ doesn't move but instead translates to next iteration. The $x_{\text {best }}$ in sub program (NM) method is the initial simplex for global optimization. The input parameter for test function will be started with $\mathrm{N}=2$, $\mathrm{m}=40$, MAXITER $=60$, LSITER $=40$, and $\delta=0.001$.

\section{NUMERICAL RESULTS}

In this section we report some numerical experiments on the $(\mathrm{EW})$ method.

Some test functions from Dixon and Szego (see [3]) are considered. In Appendix these test functions are shown. All the experiments were done on a Dell XPS 1330 laptop computer.

First we used only electromagnetic like heuristic algorithm and using the forces by (9), where the output results are called (EW1). In fact,

$F_{i}=\frac{q_{i} q_{j} e^{-a r}{ }_{i, j}}{4 \pi \varepsilon^{\circ} r_{j j}^{2}}$

where $r_{i j}=\left\|x_{j}-x_{i}\right\|$ (norm is Euclidean), and assumed :

$\varepsilon_{\circ}=8.9 \times 10^{-12}$

The (EW1) program achievement is shown in Table $\mathbf{1 .}$

To reduce the computation errors, we used $\varepsilon \circ=1$ in (9), and one can see the results in Table 2.

Since we faced long manipulations, we used electromagnetic like and electro weak heuristic methods simultaneously (EW2), and the results on the test functions are shown in Table 3, where we assumed $\varepsilon_{\circ}=1$. In Table 4, again we found the results where assumed $\varepsilon \circ=8.9 \times 10^{-12}$.

Now, we combine electromagnetic like heuristic algorithm; with attraction- repulsion force, with the Nelder-Mead 
Table 2. (EW 1) Program with $\varepsilon_{\circ}=1$

\begin{tabular}{|c|c|c|c|}
\hline $\mathbf{f}(\mathbf{x})($ Optimized $)$ & $\mathbf{f}(\mathbf{x})($ best $)$ & xbest (best) & Test Function \\
\hline 0.0000 & 0.0028 & $(1.0024,0.0173)$ & Complex \\
\hline 0.0000 & 3.5312 & $(-0.2468,3.4309)$ & Davis \\
\hline 0.0000 & 0.6731 & $(-0.6332,-9.3396)$ & Griewank \\
\hline 0.0000 & 1.4852 & $(2.9499,2.2954)$ & Himmelblau \\
\hline 0.0000 & 0.4709 & $(0.7099,0.4418)$ & Rosenbrock \\
\hline-38.85 & -38.5968 & $(12.1000,5.6268)$ & Spiky \\
\hline 0.0000 & 0.1258 & $(0.2677,0.0509)$ & Stenger \\
\hline
\end{tabular}

Table 3. (EW 2) Program with $\varepsilon_{\circ}=1$

\begin{tabular}{|c|c|c|c|}
\hline $\mathbf{f}(\mathbf{x})($ Optimized $)$ & $\mathbf{f}(\mathbf{x})($ Best $)$ & xbest $($ Best $)$ & Test Function \\
\hline \hline 0.0000 & 0.0271 & $(-0.5532,0.8591)$ & Complex \\
\hline 0.0000 & 2.3160 & $(2.7616,-2.7103)$ & Davis \\
\hline 463.0000 & 463.0800 & $(0.7481,-5.8716)$ & E1 \\
\hline 0.0000 & 0.2489 & $(-2.7462,3.9470)$ & Hiiewank \\
\hline 0.0000 & 0.1805 & $(-2.7727,3.1906)$ & Rastrigin \\
\hline-2.0000 & -1.4967 & $(0.3600,-0.3081)$ & Rosenbrock \\
\hline 0.0000 & 0.2738 & $(1.1138,1.2916)$ & Spiky \\
\hline-38.85 & -38.7328 & $(12.0995,5.7256)$ & Stenger \\
\hline 0.0000 & 0.0835 & $(0.2310,0.0575)$ & \\
\hline
\end{tabular}

Table 4. (EW 2) Program with $\varepsilon \circ=8.9 \times 10^{-12}$

\begin{tabular}{|c|c|c|c|}
\hline $\mathbf{f}(\mathbf{x})($ Optimized) & $\mathbf{f}(\mathbf{x})($ Best $)$ & xbest $($ Best $)$ & Test Function \\
\hline \hline 0.0000 & 0.0057 & $(1.0241,0.0050)$ & Complex \\
\hline 0.0000 & 0.7134 & $(-0.2028,0.2759)$ & Davis \\
\hline 463.0000 & 463.7006 & $(1.0316,6.0579)$ & E1 \\
\hline 0.0000 & 0.2166 & $(2.7535,4.6074)$ & Hriewank \\
\hline 0.0000 & 0.0735 & $(3.0482,1.9699)$ & Rastrigin \\
\hline-2.0000 & -1.6448 & $(0.0408,0.0245)$ & Rosenbrock \\
\hline 0.0000 & 0.5237 & $(0.8845,0.8538)$ & Spiky \\
\hline-38.85 & 38.6196 & $(12.1000,5.6261)$ & Stenger \\
\hline 0.0000 & 0.0302 & $(-0.0965,-0.0056)$ & \\
\hline
\end{tabular}

Table 5. Combinational Program (EW) for Test Function

\begin{tabular}{|c|c|c|c|c|}
\hline Program Iteration & $\mathbf{f}(\mathbf{x})($ Optimized $)$ & $\mathbf{f}(\mathbf{x})($ best $)$ & xbest (best) & Test Function \\
\hline 129 & 0.0000 & 0.0000 & $(0,0)$ & Davis \\
\hline 45 & 0.0000 & 0.0000 & $(0,0)$ & Griewank \\
\hline 56 & 0.0000 & $1.2314 \mathrm{e}-005$ & $(-2.8046,3.1310)$ & Himmelblau \\
\hline 68 & 0.0000 & $1.0075 \mathrm{e}-005$ & $(1.0009,1.0021)$ & Rosenbrock \\
\hline 50 & 0.0000 & 0.0000 & $(0,0)$ & Stenger \\
\hline
\end{tabular}


Table 6. A Comparison Among Different Optimization Method used for Goldenstein Price Test Function [1, 9, 10].

\begin{tabular}{|cc|}
\hline $\begin{array}{c}\text { Averaged Evaluation } \\
\text { Function GP }\end{array}$ & Method \\
\hline \hline 300 & Mod. Bremmerman \\
1495 & Gomulka - V.M. \\
2500 & Price \\
362 & Mockus \\
82 & Perttunen \\
113 & Perttunen -Stuckman \\
191 & Jones et al \\
1018 & Storn - Price \\
81 & MCS \\
420 & EM \\
60 & EW \\
\hline
\end{tabular}

heuristic searching method, the first algorithm moves the sample point from feasible region toward optimization, then with using the Nelder-Mead method, the former points in electromagnetic like algorithm, converges toward global optimization. We called the combined algorithm as (EW) and the results on test functions with this new method are shown in Table 5, and Table $\mathbf{6}$.

First column in Table- 6 shows the average evaluation of goldenstein price (GP) function obtained from mentioned above different methods, where with (EW) optimization method only with 60 iteration reached to optimization. This least iteration to reach optimization in comparison with other methods is the best privilege of the global heuristic optimization method we called (EW) method.

\section{Privilege of the (EW) Heuristic Global Optimization}

1. Reduced time of optimization calculation.

2. Reduced error of global optimization so as for most test functions the (EW) method lead to precise answer while the math error vanishes.

3. The method converges very fast to the global optimization.

4. The method is usable even for irregular objective functions that are non-differentiable in some points.

\section{CONCLUSION REMARKS}

The combination (EW) method devised and presented here is for global optimization bounded functions.

The (EM) and (EW) methods are based upon electrostatic attraction and repulsion forces of charged particles.

It is assumed that each charged point surrounded the global optimization region started to search global optimization afterwards (NM) simplex is used to obtain best global optimization.

This combinational method used to find global optimization of test functions obtained from [3, 4] and [10] which show the accuracy of the method. The results presented satisfactory achievements even for defined functions with frequent local optimization, so as reaching to global optimization completely efficient even if these are not differentiable functions.

Noticeably for nonlinear and unbounded problems the research should be continued.

\section{APPENDIX}

Here the used test functions are introduced.

\section{(i) Complex}

$$
\begin{aligned}
& f(x)=\left(x_{1}^{3}-3 x_{1} x_{2}^{2}-1\right)^{2}+\left(3 x_{1}^{2} x_{2}-x_{2}^{3}\right)^{2} \\
& -2 \leq x_{i} \leq 2 \mathrm{i}=1,2 \\
& x^{*}=\left\{(1,0),\left(\frac{-1}{2}, \sqrt{\frac{1}{2}}\right)\right\} \\
& f\left(\mathrm{x}^{*}\right)=0
\end{aligned}
$$

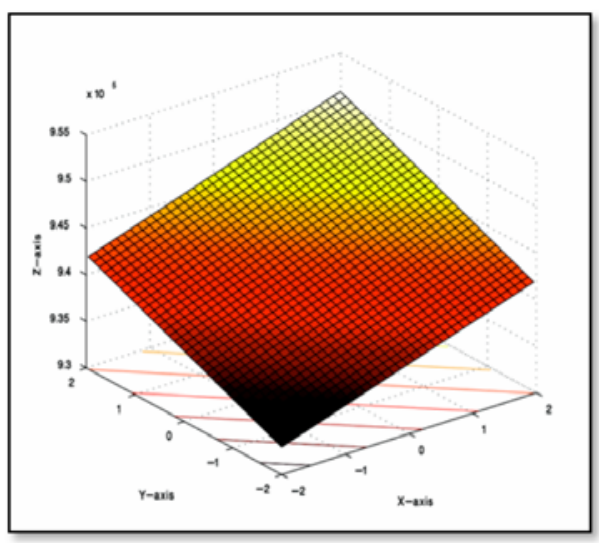

(ii) Davis

$$
\begin{aligned}
& f(x)=\left(x_{1}^{2}+x_{2}^{2}\right)^{0.25}\left[\sin ^{2}\left(50\left(3 x_{1}^{2}+x_{2}^{2}\right)^{0.1}\right)+1\right] \\
& -100 \leq x_{i} \leq 100 \quad i=1,2 \\
& x^{*}=\{(0,0)\}
\end{aligned}
$$

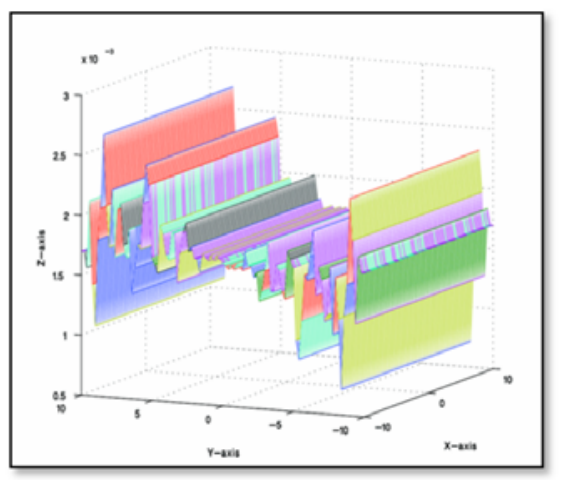

$f\left(x^{*}\right)=0$

(iii) $\mathrm{E} 1$

$$
\begin{aligned}
& f(x)=x_{1}^{2}-2 x_{1}+x_{2}^{2}+12 x_{2}+500 \\
& -10 \leq x_{i} \leq 10 \quad i=1,2
\end{aligned}
$$


$x^{*}=\{(1.0022,5.9968)\}$

$f\left(x^{*}\right)=463.0000$

(iv) Goldstein and Prices (GP)

$$
\begin{aligned}
f(x)=(1+ & \left(x_{1}+x_{2}+1\right)^{2}\left(19-14 x_{1}+3 x_{1}^{2}-14 x_{2}+6 x_{1} x_{2}\right. \\
& \left.\left.+3 x_{2}^{2}\right)\right) \cdot\left(30+\left(2 x_{1}-3 x_{2}\right)^{2}\left(18-32 x_{1}+12 x_{1}^{2}+48 x_{2}\right.\right. \\
& \left.\left.-36 x_{1} x_{2}+27 x_{2}^{2}\right)\right)
\end{aligned}
$$

$-2 \leq x_{i}<2 \quad i=1,2$

$x^{*}=\{(0,-1)\}$

$f\left(x^{*}\right)=3$

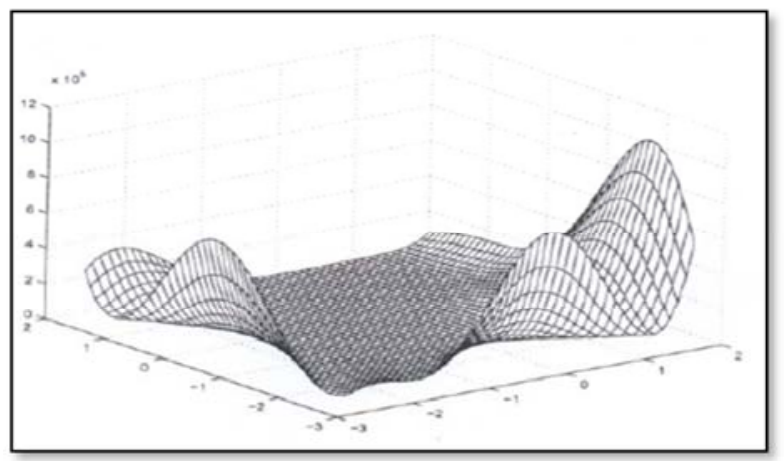

(v) Griewank

$f(x)=\frac{1}{200}\left(x_{1}^{2}+x_{2}^{2}\right)-\cos x_{1} \cos \frac{x_{2}}{\sqrt{2}}+1$

$-100 \leq x_{i} \leq 100 \quad i=1,2$

$x^{*}=\{(0,0)\}$

$f\left(x^{*}\right)=0$

\section{(vi) Himmelblau}

$$
\begin{aligned}
& f(x)=\left(x_{1}^{2}+x_{2}-11\right)^{2}+\left(x_{1}+x_{2}^{2}-7\right)^{2} \\
& -6 \leq x_{i} \leq 6 \quad i=1,2 \\
& x^{*}=\left\{\begin{array}{c}
(3,2),(-2.805118,3.131312),(3.584428,-1.848126) \\
,(-3.779310,-3.283186)
\end{array}\right\}
\end{aligned}
$$

$f\left(x^{*}\right)=0$

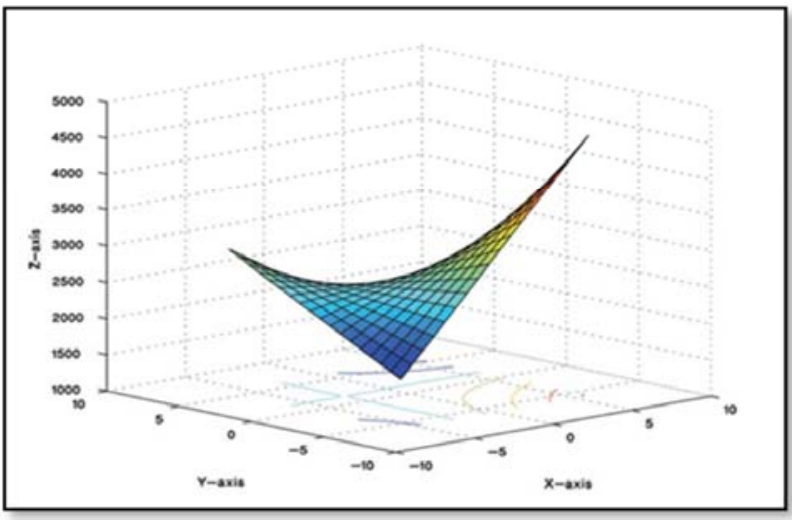

(vii) Rastrigin

$$
\begin{aligned}
& f(x)=x_{1}^{2}+x_{2}^{2}-\cos \left(18 x_{1}\right)-\cos \left(18 x_{2}\right) \\
& -5.12 \leq x_{i} \leq 5.12 \quad i=1,2 \\
& x^{*}=\{(0,0)\} \\
& f\left(x^{*}\right)=-2
\end{aligned}
$$

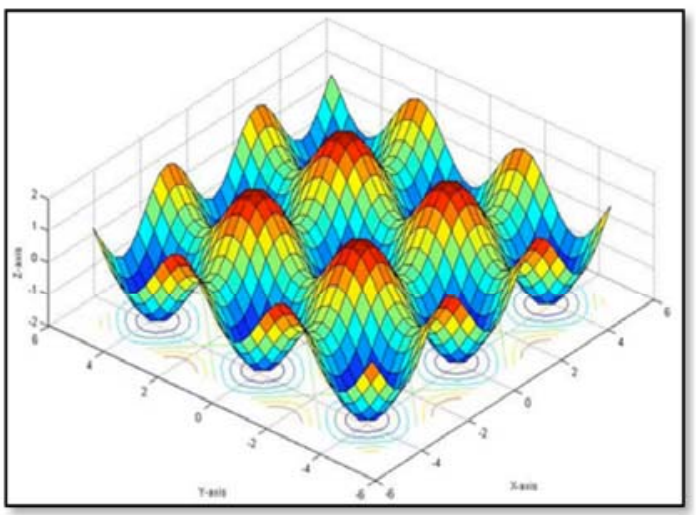

(viii) Rosenbrok $\left(R_{n}\right)$

$$
\begin{aligned}
& f_{n}(x)=\sum_{i=1}^{n-1}\left[100\left(x_{i}^{2}-x_{i+1}\right)^{2}+\left(x_{i}-1\right)^{2}\right] \\
& -5 \leq x_{i} \leq 10 \quad i=1,2, \ldots, n \\
& x^{*}=\{(1, \ldots, 1)\} \\
& f_{n}\left(x^{*}\right)=0
\end{aligned}
$$

(ix) Stenger

$$
\begin{aligned}
& f(x)=\left(x_{1}^{2}-4 x_{2}\right)^{2}+\left(x_{2}^{2}-2 x_{1}+4 x_{2}\right)^{2} \\
& -1 \leq x_{i} \leq 4 \quad i=1,2 \\
& x^{*}=\{(0,0),(1.695415,0.7186082)\} \\
& f\left(x^{*}\right)=0
\end{aligned}
$$

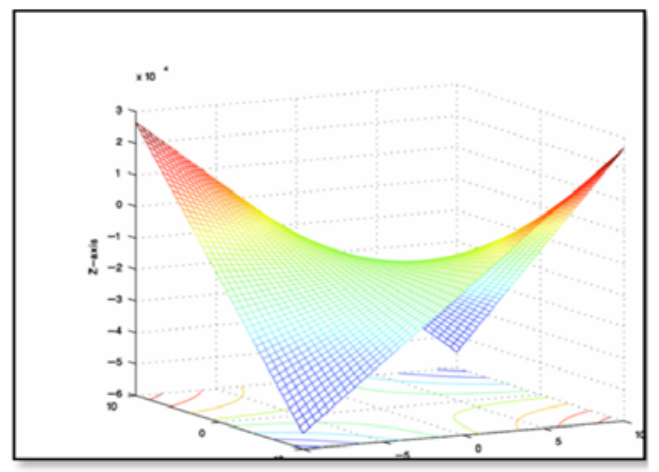

\section{CONFLICT OF INTEREST}

None declared.

\section{ACKNOWLEDGEMENT}

None declared.

\section{REFERENCES}

[1] C. G. E. Boender, A. H. G. R. Kan, and G. T. Tinimer, "A Stochastic approach to global optimization", Comput. Math. Prog. (NATOASI Series), vol. F15, pp. 291-308, 1985. 
[2] M. Demirhan, L. Ozdamar, L. Hevacioglu, and S. I. Birbil, "A geometric partitioning metaheuristic for global optimization", $J$. GLOBAL. OPTIM., vol. 14, pp. 415-435, 1999.

[3] L. C. W. Dixon, and G. P. Szego, "The Global Optimition problem: an introduction Towards Global Optimization 2. North-holland, Amesterdam", pp. 1-15, 1978.

[4] C. B. Garcia, and W. I. Zangwill, "Path ways to Solutions, Fixed Points and Equilibria". Prentice-Hall: Englewoods Cliffs, NJ, 1981.

[5] J. D. Pinter, "Global Optimization in Action, Continuous and Lipschitsz Optimization: Algorithms, Implementations And Applications, Volume 6 Of Noneconvex Optimization and Its Applications", Kluwer Academic Publishers: Dodrecht, The Nethelands, 1996.

[6] P. M. Pardalos, and J. B. Rosen, "Methods for Global Concave Minimization", Bilbliogr. Surv. SIAM Rev., vol. 28, pp. 367-379, 1986.

[7] P. Brandimarte, "Numerical methods in finance and economies: A MATLAB ${ }^{\circledR}$-Based Introduction”, John Wiley \& Sons, Inc.: NJ, 2006.
[8] T. Klastorin, "Critical Path Method (CPM) is a mathematically based algorithm for scheduting a set of project activities", it is an important tool for effective project management. In: Project Management Tools and Trade-off, $3^{\text {rd }}$ ed. John Wiley \& Sons, Inc.: NJ, 2003.

[9] S. I. Birbil, and S. C. Fang, "An Electromagnetism-like Mechanism for Global Optimization", J. GLOBAL. OPTIM., vol. 25, pp. 263-282, 2003.

[10] E. Ansari, "The Combination of Heuristic (EM) and (NM) Methods for Global Optimization". M.Sc. Dissertation. Islamic Azad University, Mashhad Branch, Iran, 2009.

[11] G. R.Walsh, Methods of Optimization, John Wiley and Sons: NJ, 1975.

[12] F. Gao, and L. Han, "Implementing the Nelder-Mead Simplex algorithm with adaptive parameters". Comput. Optim. Appl., vol. 51, pp. 259-277, 2012

[13] S. S. Rao, Engineering Optimization: Theory and Applications, Wiley and Sons Limited: Austrailia 1996.

(C) Emami et al.; Licensee Bentham Open.

This is an open access article licensed under the terms of the Creative Commons Attribution Non-Commercial License (http://creativecommons.org/licenses/by-nc/3.0/) which permits unrestricted, non-commercial use, distribution and reproduction in any medium, provided the work is properly cited. 